\title{
Optically guided mode study of nematic liquid crystal alignment on a zero-order grating
}

\author{
B. T. Hallam* and J. R. Sambles \\ Thin Film Photonics, School of Physics, University of Exeter, Stocker Road, Exeter EX4 4QL, United Kingdom
}

(Received 23 November 1999)

\begin{abstract}
The characterization of a liquid crystal cell, which comprises one zero-order (that is, at the wavelength of study it is nondiffractive) diffraction grating and one rubbed polyimide-coated substrate, has been performed using an optically guided mode technique. The cell is filled with nematic liquid crystal E7 (manufactured and sold by Merck, Poole, U.K.). The excitation of fully leaky guided modes within the liquid crystal layer has allowed the optical director profile to be quantified under the application of weak in-plane electric fields. The fitting of angle-dependent optical data to multilayer optical theory yields the accurate twist profile of the liquid crystal for different field strengths. Comparisons with profiles predicted from elastic continuum theory, assuming a Rapini-Papoular-type anchoring at the surfaces, allow both the azimuthal anchoring strength at each surface and the twist elastic constant of the bulk to be accurately determined. Repeating these measurements as a function of temperature allows the surface and bulk order parameters of the grating-aligned liquid crystal to be deduced.
\end{abstract}

PACS number(s): 42.70.Df, 42.40.Eq, 42.79.Gn

\section{INTRODUCTION}

A model for the alignment of liquid crystal directors on a periodically modulated surface was first proposed by Berreman [1], who sought an explanation for the liquid crystal alignment on replicated surfaces. Since the replica had not been statically charged by rubbing, he hypothesized that the mechanism for alignment must be topological in nature, resulting from the minimization of the free energy associated with the directors lying parallel to the grating grooves compared to those lying across them. In the years following the publication of this work there was some interest in the use of gratings as an alignment layer [2-6]. Good contrast ratios in twisted nematic geometries [5,7] suggested a promising future; however, the complexity of manufacturing it as compared to using conventional rubbed polymer techniques proved to be prohibitive.

Recent advances in the high quality replication techniques of plastic films and new grating-aligned devices offering excellent viewing-angle characteristics [8] and bistability [9] have renewed interest in the use of diffraction gratings in combination with liquid crystals. According to Berreman, the use of gratings as an alignment layer offers unprecedented control over the degree of azimuthal (in-plane) anchoring energy that the surface exhibits over the liquid crystal directors. The depth and pitch of the gratings determine this anchoring energy, both of which may be accurately controlled during the manufacturing process. Although Berreman's theory assumes very shallow gratings, studies have shown that sufficiently deep gratings are also of interest, causing the suppression of an orientational transition of the directors from homogeneous to homeotropic with decreasing temperature [10]. Recent work is divided on the validity of Berreman's simple expression: some show good agreement [11], whereas others demonstrate poor agreement [12]. The authors of what is possibly the first comprehensive study in-

\footnotetext{
*FAX: 44-1392-264111.
}

Electronic address: bthallam@exeter.ac.uk volving different grating depths and pitches have invoked surface memory effects to show good agreement when a higher temperature elastic constant is employed [13].

In any device that utilizes a grating for topological alignment, the diffractive nature of the surface may be a nuisance, since it may give undesired loss of light intensity into diffracted orders and add increased complexity to device modeling. If the grating is made with a pitch less than half that of the wavelength of the radiation in the adjacent dielectric, it is nondiffractive. Any such zero-order diffraction grating exhibits form birefringence [14] and, since the feature size is small, may be modeled optically as a planar, weakly uniaxial layer.

In this work, an optically guided mode [the fully leaky guided mode (FLGM) [15]] study of a homogeneously aligned liquid crystal cell is presented, in which alignment is provided on one surface by rubbed polyimide and on the other by a zero-order diffraction grating manufactured in photoresist. The two substrates have been arranged so that the rubbing direction of the polyimide is in the same direction as the grating grooves, and the cell is filled with nematic liquid crystal E7 (manufactured by Merck, Poole U.K.). Azimuthal anchoring strengths $\left(W_{a}\right)$ of the two surfaces are determined by comparing perturbations to the optical director profile, brought about by the application of weak in-plane electric fields, to predictions of continuum elasticity theory. This method also allows the twist elastic constant $\left(k_{22}\right)$ to be deduced. All of the director profiles are accurately determined by fitting angle-dependent reflectivity and transmission data to multilayer optical theory. Measurements have been conducted at a series of temperatures up to the clearing temperature of the nematic liquid crystal. By a careful analysis of all the results, both the surface and bulk order parameters are obtained. As far as we are aware, this work represents the first time that both the bulk and surface order parameters of a grating-aligned liquid crystal cell have been determined from the same measurements.

\section{THEORY}

Although the mathematical treatment is outlined here, a full description may be found elsewhere [16]. The twist de- 
formation induced in a nematic liquid crystal layer via an in-plane electric field is governed by the Euler-Lagrange equation associated with the change in the free energy of the nematic, subject to the in-plane field $E$ :

$$
\frac{d^{2} \phi}{d z^{2}}=-\frac{\varepsilon_{o} \Delta \varepsilon E^{2}}{k_{22}} \sin \phi \cos \phi,
$$

where $\phi=\phi(z)$ is the twist angle of the nematic liquid crystal $z$ is the direction through the cell thickness, and $\Delta \varepsilon$ is the dielectric anisotropy of the material.

This equation can be solved for the boundary conditions supplied by the torque-balance equations assuming finite surface anchoring (where the anchoring strength on the top surface, $W_{a 1}$, does not have to equal the anchoring strength on the bottom surface, $W_{a 2}$ ):

$$
\begin{gathered}
k_{22}\left(\frac{d \phi}{d z}\right)_{z=d / 2 \phi=\phi_{d 1}}=2 W_{a 1}\left(\phi_{o 1}-\phi_{d 1}\right), \\
k_{22}\left(\frac{d \phi}{d z}\right)_{z=-d / 2 \phi=-\phi_{d 1}}=2 W_{a 2}\left(\phi_{o 2}-\phi_{d 2}\right),
\end{gathered}
$$

and a zero-field pretwist in the cell:

$$
\begin{aligned}
& k_{22}\left(\frac{\phi_{o 1}^{\prime}+\phi_{o 2}^{\prime}}{d}\right)=2 W_{a 1}\left(\phi_{o 1}-\phi_{o 1}^{\prime}\right), \\
& k_{22}\left(\frac{\phi_{o 1}^{\prime}+\phi_{o 2}^{\prime}}{d}\right)=2 W_{a 2}\left(\phi_{o 2}-\phi_{o 1}^{\prime}\right),
\end{aligned}
$$

where $\phi_{o 1}$ and $-\phi_{o 2}$ are the easy axes of alignment, $\phi_{o 1}^{\prime}$ and $-\phi_{o 2}^{\prime}$ are the actual twist angles of the directors at each surface, $\phi_{d 1}$ and $-\phi_{d 2}$ are the surface twist angles under application of the electric field, and $d$ is the cell thickness.

Hence if $\Delta \varepsilon$ is known, together with the magnitude of the applied electric field, the zero-field surface twist angles, and the cell thickness, then choosing values of the constants $k_{22}$, $W_{a 1}$, and $W_{a 2}$ allows the twist profile of the director through the cell to be generated. A multilayer optical theory, based on a scattering matrix [17] is then used to generate angledependent optical data from this profile. These serve as model predictions for several different electric field strengths $(E)$. The magnitude of $E$ can be calculated from Eq. (6):

$$
E=\frac{2 V}{\pi g}
$$

in which $V$ is the voltage and $g$ is the electrode gap.

\section{EXPERIMENTAL DETAILS}

Figure 1 shows the cell geometry used in this work. Standard index glass prisms are optically matched onto the cell by a suitably mixed silicone oil index matching fluid. The entire assembly is then placed on the center of rotation of a rotating table. The prisms allow incident laser light to be coupled into guided modes within the liquid crystal layer. Since both the liquid crystal refractive indices are higher than the surrounding glass, these modes are able to "leak"
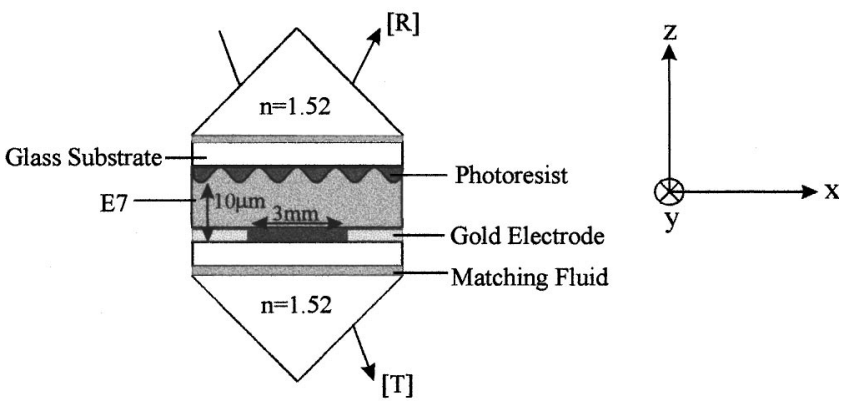

FIG. 1. A slice (in the incidence plane) through the experimental cell geometry. Alignment of the liquid crystal is homogeneous and close to parallel to the electrode edges. Standard-index glass prisms are optically matched to the cell by a silicone oil matching fluid.

into the incident and transmitted half spaces, thus appearing as broad features in angle-dependent reflectivity and transmission data. Polarizers allow the incident and detected polarization states to be set. Thus a set of eight data sets may be recorded to check the validity of any proposed model. These comprise, for reflection $(R)$ and transmission $(T): R_{p p}, R_{p s}$, $R_{s p}, R_{s s}, T_{p p}, T_{p s}, T_{s p}$, and $T_{s s}$, where the first subscript denotes the incident polarization and the second subscript denotes the recorded polarization.

Gold electrodes (350 $\mathrm{nm}$ thick) with a 3-mm electrode gap were evaporated onto the bottom substrate to allow the application of a $10-\mathrm{kHz}$ ac electric field within the plane of the substrate. Polyimide was spin-coated onto the substrate at 3500 revolutions per second $\left(\mathrm{rev} \mathrm{s}^{-1}\right)$ for $35 \mathrm{~s}$ before being ovenbaked at $275^{\circ} \mathrm{C}$ for $3.5 \mathrm{~h}$. A velvet-covered rolling drum was used to carefully rub the polyimide in a direction parallel to the electrode edges.

The superstrate was prepared by spin deposition of photoresist (Shipley, 1805) at $3000 \mathrm{rev} \mathrm{s}^{-1}$ for $60 \mathrm{~s}$. A zero-order diffraction grating with an area of $2 \times 2 \mathrm{~cm}$ was prepared in the photoresist by using standard interferographic techniques [18] involving oblique exposure of a helium-cadmium $\left(\lambda_{0}\right.$ $=325-\mathrm{nm}$ ) laser. The grating was made robust by baking at $180^{\circ} \mathrm{C}$ for $14 \mathrm{~h}$. A grating pitch of $176.2 \mathrm{~nm}$ was determined from a Littrow-angle scan of the grating in the ultraviolet. To determine the depth of the grating, it was placed normal to the incident plane between two crossed polarizers with the grating grooves orientated at an angle of $45^{\circ}$ to the transmission axis of the incident polarizer. The magnitude of the light transmitted through the system was then recorded. As previously mentioned, zero-order diffraction gratings exhibit from birefringence. This arises because linearly polarized incident radiation experiences a different effective-refractive index when its electric vector is orientated parallel to the grating grooves than when it is orientated perpendicular to them [14]. This birefringence induces changes in the polarization of linearly polarized light transmitted through the grating. It is a maximum for normally incident light polarized at $45^{\circ}$ to the grating grooves. Since the degree of change in polarization is dependent on the thickness of the birefringent material through which the light has passed (the depth of the grating), it may be employed to provide a measure of the depth of the grating grooves. Measurements of this type determined the depth of the grating to be $62 \pm 8 \mathrm{~nm}$ [19]. Figure 2 is a scanning electron micrograph of a similar surface and shows the 


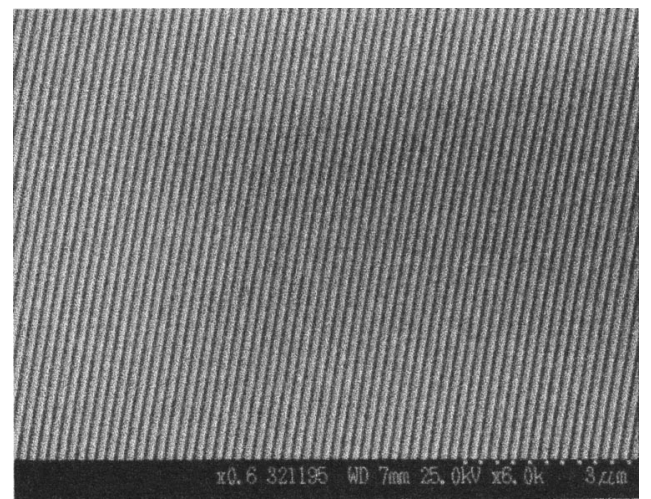

FIG. 2. A scanning electron micrograph of an interferographically produced grating in photoresist. The grating pitch is $174.6 \mathrm{~nm}$ and is imaged from overhead, demonstrating the uniformity and large-area $(2 \times 2 \mathrm{~cm})$ coverage of the surface produced.

uniformity and large area coverage of the manufactured gratings.

Approximately parallel (nontwisted) alignment of the liquid crystal directors was achieved by suitable orientation of the substrate with respect to the superstrate (the groove direction was carefully marked during the manufacturing process and the polyimide was rubbed in the direction of the electrode edges). The true director twist through the system was subsequently obtained by fitting FLGM optical data with no field applied. Beaded glue provided a plate spacing of approximately $6 \mu \mathrm{m}$ and the liquid crystal was introduced into the cell under vacuum in the isotropic phase. Subsequently, it was slowly cooled into the nematic phase to ensure a good monodomain. Illumination of the cell was provided by light from a HeNe $\left(\lambda_{0}=632.8-\mathrm{nm}\right)$ laser and the beam spot was controlled to approximately $0.5 \mathrm{~mm}^{2}$.

Reflectivity and transmission data were recorded as a function of the angle of incidence for set polarizations with different electric field strengths applied $\left(E_{\max }<3.2\right.$ $\left.\times 10^{-2} \mathrm{~V} \mu \mathrm{m}^{-1}\right)$. Fitting this data to predictions from multilayer optical theory gives the zero-field director profile, including the true zero-field twist angles and the cell thickness. Using these data, in conjunction with measured values of the material's dielectric anisotropy at $10 \mathrm{kHz}$ [16], allows field-induced director profiles generated by a continuum model to be established. Careful analysis of the sensitive polarization conversion signal in transmission $\left(T_{p s}\right)$ yields both the twist elastic constant $k_{22}$ and the anchoring energies at each substrate, $W_{a 1}$ and $W_{a 2}$. Such fits were repeated at several temperatures.

\section{RESULTS}

To reduce the complexity of computer modeling the zeroorder grating surface in detail, the photoresist grating and interfacial liquid crystal were divided into two discrete planar layers. The first of these layers is isotropic to represent bulk photoresist, and the other is anisotropic (with refractive indices intermediate to those of the liquid crystal and the photoresist), representing the hybrid grating/liquid crystal interfacial layer. This is a fair assumption since the grating features are necessarily small (groove widths $\sim 88 \mathrm{~nm}$ ), and the grating does not introduce diffraction into the optics. To

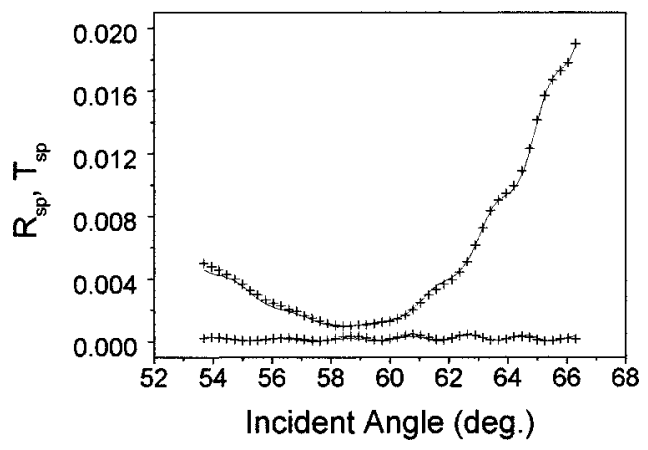

FIG. 3. Typical fits (solid lines) to angle-dependent polarizationconversion data (crosses).

ensure an unprejudiced fit was obtained, a genetic algorithm was employed to search the "parameter hyperspace" for the model prediction that gives the least sum of squares error for the set of eight data sets being simultaneously fitted. To further improve the sum of squares error a "hill-climbing", minimization program was then executed.

Figure 3 shows typical fits to angle-dependent optical data. The theory is shown as a solid line and the experimental data as crosses. The polarization conversion data sets $R_{s p}$ and $T_{s p}$ are plotted and have been fitted to the same director profile. To generate these fits, the resulting model director profile comprised a twist through the cell of $0.96 \pm 0.05^{\circ}$ and a linearly graded tilt with surface tilt angles of 88.67 $\pm 0.08^{\circ}$ and $87.61 \pm 0.08^{\circ}\left(90^{\circ}\right.$ is in the substrate plane) at the polyimide and photoresist surfaces, respectively. This model was not found to change appreciably with temperature. Using this zero-field profile in the continuum model provides the fits, at a temperature of $32{ }^{\circ} \mathrm{C}$, to the transmitted polarization conversion signal $T_{p s}$ for several different electric field strengths shown in Fig. 4. Again the data are shown as crosses and the theory as a solid line.

Fitting these data at several temperatures yields the values, for E7 on rubbed polyimide and a zero-order diffraction grating in photoresist, of $W_{a}$ and $k_{22}$ shown in Figs. 5(a) and 5 (b). These values for $k_{22}$ are in good agreement with those obtained from light scattering experiments [20]. The clearing temperature for E7 is about $60^{\circ} \mathrm{C}$; however, data have only been collected to a maximum temperature of $57.1^{\circ} \mathrm{C}$, above

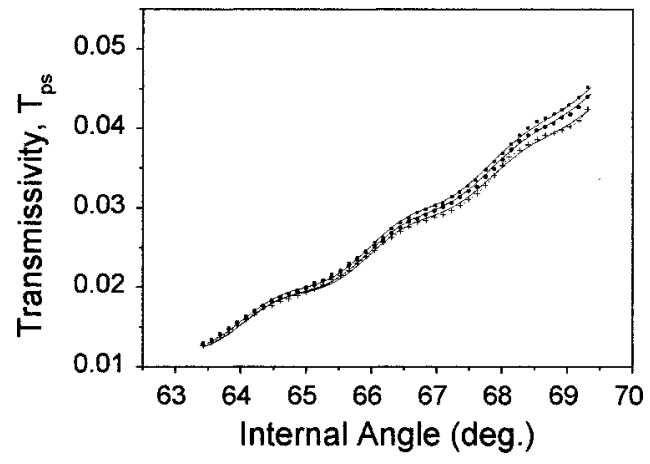

FIG. 4. The effect of an in-plane ac field $\left(0 \mathrm{~V}, 100 \mathrm{~V}, 140 V_{\mathrm{RMS}}\right.$ across a 3-mm electrode gap) on the transmission signal $T_{\mathrm{ps}}$ at $32{ }^{\circ} \mathrm{C}$. Experimental data are shown as crosses and the theory as a solid line. 

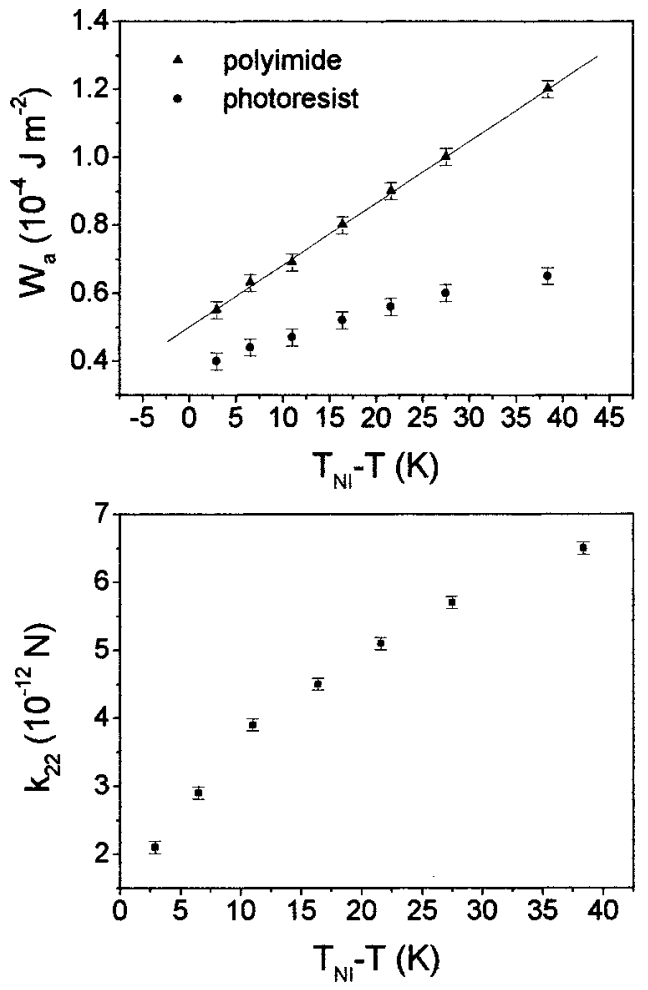

FIG. 5. (a)The azimuthal anchoring strength, as a function of temperature, of nematic liquid crystal E7 on a photoresist grating and on a rubbed polymer film. The solid line is a fit to Eq. (8) with $b=\left(0.1822 \times 10^{-6}\right) \mathrm{J}^{1 / 2} \mathrm{~m}^{-1} \mathrm{~K}^{-1 / 2}$. (b) The twist elastic constant of nematic liquid crystal E7 as a function of temperature.

which the material was observed to enter a biphasic region, thus prohibiting the collection of suitable optical data.

\section{DISCUSSION}

Figure 5(a) shows a linear temperature dependence of $W_{a}$ on the polyimide surface. This behavior may be understood by allowing for variations in the order parameter close to the nematic-wall interface [21]. In [22], the following functional form for the surface order parameter $\left(Q_{s}\right)$ has been derived:

$$
Q_{s}=a+b\left(T_{N I}-T\right)^{1 / 2},
$$

where $a$ and $b$ are positive constants.

Equations (4) and (5) show that $W_{a}$ is expected to be directly proportional to $k_{22}$. This is confirmed in Fig. 6 for temperatures sufficiently far from the clearing temperature. Generally speaking, the effective elastic constant $k$ is also proportional to the square of the order parameter; hence we may assume that close to the polyimide surface

$$
W_{a} \propto Q_{s}^{2}=\left[a+b\left(T_{N I}-T\right)^{1 / 2}\right]^{2} .
$$

Thus sufficiently far from $T_{N I}, W_{a}$ is linear with temperature. The experimentally obtained value of $b$ is $(1.822$ $\left.\times 10^{-6}\right) \mathrm{J}^{1 / 2} \mathrm{~m}^{-1} \mathrm{~K}^{-1 / 2}$.

According to Berreman, the temperature dependence of the anchoring on the grating should vary as the temperature dependence of the elastic constant. Figure 6 is a plot of $\log _{10}\left(W_{a}\right)$ against $\log _{10}\left(k_{22}\right)$ for both surfaces. The graph shows that, up to about $50^{\circ} \mathrm{C}$, the anchoring energy on the

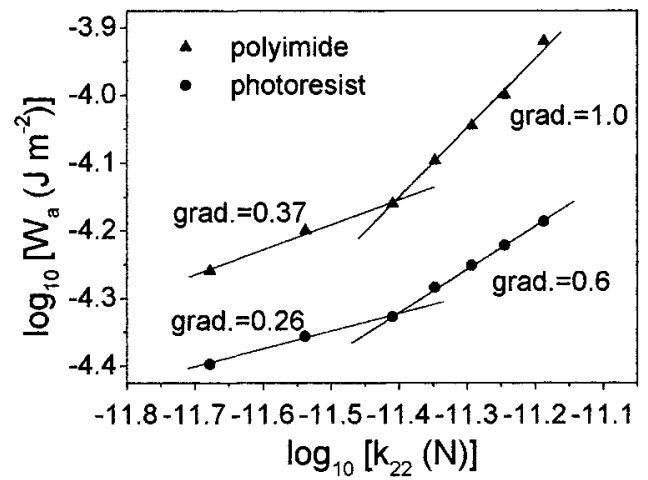

FIG. 6. A plot of $\log _{10}\left(W_{a}\right)$ against $\log _{10}\left(k_{22}\right)$ shows that (at lower temperatures) the anchoring strength of the surface is directly proportional to the bulk elastic constant in the case of polyamide, but not for the grating surface.

polyimide surface is proportional to the elastic constant as previously stated. However, this is not the case for the grating surface where a slope of 0.6 is found. Further consideration of this anomaly suggests that the curvature (and hence the strain energy density) associated with deep grating grooves will result in a local surface elastic constant that will be different from that in the bulk. The anomaly may provide direct evidence for this fact. Indeed, the values calculated for $W_{a}$ using the Berreman model are all larger than those determined experimentally. However, in agreement with [13], reanalyzing these data with a high temperature $\left(\sim 55^{\circ} \mathrm{C}\right)$ elastic constant brings the results into almost exact agreement. This "surface" value of $k_{22}$ is nearly temperature independent (the values of $k_{22}$ used to bring the Berreman predictions in line with the experimental results correspond to changing the required surface temperature by $<2{ }^{\circ} \mathrm{C}$ ), which supports the surface memory hypothesis of [13].

Both curves in Fig. 6 appear to change gradient for temperatures greater than about $50{ }^{\circ} \mathrm{C}$. We believe that this is the result of the surface order parameter, which is less than that of the bulk [6] at room temperatures (but which does not have such a strong temperature dependence), playing an increasingly important role as the bulk order decreases rapidly with increasing temperature (Fig. 7 and 8).

Using the relationship in Eq. (8), the surface order parameters for the two different alignment layers have been deduced from the experimental measurements of the azimuthal

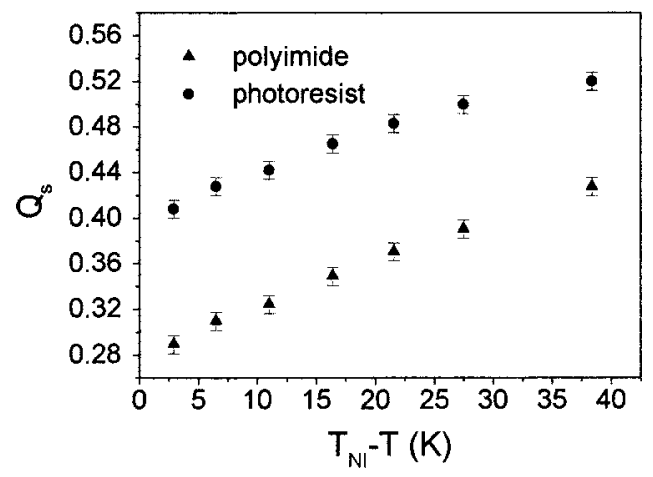

FIG. 7. Scalar surface order parameters $\left(Q_{s}\right)$ for liquid crystal alignment by a short-pitch grating and by rubbed polyimide as a function of temperature. 


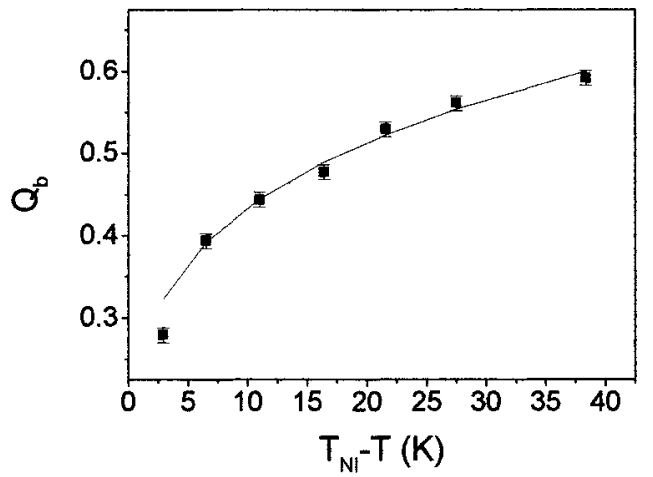

FIG. 8. The scalar bulk order parameter $Q_{b}$ as a function of temperature. Experimental data are shown as squares and $Q_{b}$ $=0.25\left(T_{N I}-T\right)^{0.24}$ is shown as a solid line.

anchoring energy. The results have been normalized by extrapolation to $0 \mathrm{~K}$, at which temperature the liquid crystal is assumed to be perfectly ordered $\left(Q_{s}=1.0\right)$, and are plotted in Fig. 7. Extrapolation of these curves to the nematicisotropic phase transition would suggest a nonzero order parameter at the surfaces. Indeed, the two plots display a weak dependence on temperature, indicating that these surface regions have an almost "frozen', order and behave very differently to the bulk.

Since the FLGM technique allows determination of the liquid crystal refractive indices, the bulk order parameter may be evaluated using the Vuks formula [23]. Denoting $n_{e}$ and $n_{o}$ as the refractive indices parallel and perpendicular to the optical director respectively, the Vuks relationship for a uniaxial material may be written as

$$
Q_{b}=\frac{n_{e}^{2}-n_{o}^{2}}{n_{\mathrm{av}}^{2}-1} \frac{\alpha_{\mathrm{av}}}{\Delta \alpha},
$$

where

$$
n_{\mathrm{av}}^{2}=\left(\frac{n_{e}^{2}+2 n_{o}^{2}}{3}\right) \text {. }
$$

The ratio of the average molecular polarizability $\left(\alpha_{\mathrm{av}}\right)$ to the anisotropy of molecular polarizability $(\Delta \alpha)$ may be determined from a Haller plot [24], which assumes that $Q_{b}=1.0$ at $0 \mathrm{~K}$. The experimentally determined value for $\alpha_{\mathrm{av}} / \Delta \alpha$ is $1.26 \pm 0.02$. Figure 8 shows a plot of the bulk order parameter as a function of temperature. Unlike the surface regions, the bulk displays a strong temperature dependence. The solid line is a plot of $Q_{b}=0.25\left(T_{N I}-T\right)^{0.24}$, the exponent of which was determined from a plot of $\log _{10}\left(Q_{b}\right)$ against $\log _{10}\left(T_{N I}-T\right)$. As mentioned in previous work [16], we do not assume that this exponent is experimental evidence for tricritical behavior in E7 since the measurements have been taken at temperatures beyond the validity range of the Landau theory.

\section{CONCLUSIONS}

This work presents what we consider the first study of a zero-order diffraction grating aligned liquid crystal cell. A complete optical characterization of the cell has been performed using the fully leaky guided mode technique. Twist distortions in the liquid crystal director profile have been induced via weak in-plane electric fields and analyzed by fitting angle-dependent reflectivity and transmission data to multilayer optical theory. Comparisons of these distortions with those predicted from an elastic continuum theory have yielded the azimuthal anchoring energy of the cell and the twist elastic constant of the liquid crystal. The azimuthal anchoring energy of the grating surface probably arises from a combination of topological effects and adsorption of the liquid crystal molecules into the polymer surface. These results have been discussed in terms of the Berreman theory, and surface memory effects.

The measurements have been performed at a series of different temperatures, allowing the surface and bulk order parameters of a grating aligned liquid crystal cell to be independently determined from the same cell measurements. These results have been analyzed and their implications discussed.

An interfacial region of reduced order (in comparison with the bulk) has been found at the cell surfaces at room temperature. The surface order is found to be larger for alignment on a photoresist grating than for a rubbed polyimide surface. Although this result may be due to topological effects, it could also be due to the different treatments the two polymers have received. Rubbing is a harsh process and may introduce a good deal of roughness on a molecular level, therefore preventing better surface order. While the surface order only shows a weak dependence on temperature, the bulk order is seen to decrease rapidly with increasing temperature, becoming less ordered than the surface as the isotropic phase is approached.

\section{ACKNOWLEDGMENTS}

The authors gratefully acknowledge the financial support of the Engineering and Physical Sciences Research Council and the Defense Evaluation and Research Agency (Malvern).
[1] D. W. Berreman, Phys. Rev. Lett. 28, 1683 (1972).

[2] D. C. Flanders, D. C. Shaver, and H. I. Smith, Appl. Phys. Lett. 32, 112 (1978).

[3] J. Cheng and G. D. Boyd, Appl. Phys. Lett. 35, 444 (1979).

[4] A. Sugimura, N. Yamamoto, and T. Kawamura, Jpn. J. Appl. Phys. 20, 1343 (1981).

[5] Y. Kawata, K. Takatoh, M. Hasegawa, and M. Sakamoto, Liq. Cryst. 16, 1027 (1994).
[6] G. P. Bryan-Brown, J. R. Sambles, and K. R. Welford, Liq. Cryst. 13, 615 (1993).

[7] M. Nakamura and M. Ura, J. Appl. Phys. 52, 210 (1981).

[8] G. P. Bryan-Brown, C. V. Brown, I. C. Sage, and V. C. Hui, Nature (London) 392, 365 (1998).

[9] G. P. Bryan-Brown, C. V. Brown, J. C. Jones, E. L. Wood, I. C. Sage, P. Brett, and J. Rudin, SID J. 28, 37 (1997).

[10] H. V. Känel, J. D. Litster, J. Melngailis, and H. I. Smith, Phys. 
Rev. A 24, 2713 (1981).

[11] C. J. Newsome, M. O’Neill, R. J. Farley, and G. P. BryanBrown, Appl. Phys. Lett. 72, 2078 (1998).

[12] R. Barberi, I. Dozov, M. Giocondo, M. Iovane, Ph. MartinotLagarde, D. Stoenescu, S. Tonchev, and L. V. Tsonev, Eur. Phys. J. B 6, 83 (1998).

[13] E. L. Wood, G. W. Bradberry, P. S. Cann, and J. R. Sambles, J. Appl. Phys. 82, 2483 (1997).

[14] M. Born and E. Wolf, Principles of Optics (Permagon, New York, 1980).

[15] F. Yang and J. R. Sambles, J. Opt. Soc. Am. B 16, 488 (1999).

[16] B. T. Hallam, J. R. Sambles, and C. V. Brown, J. Appl. Phys. 86, 6682 (1999).

[17] D. Y. K. Ko and J. R. Sambles, J. Opt. Soc. Am. A 5, 1863
(1988).

[18] M. C. Hutley, Diffraction Gratings (Academic, London, 1982).

[19] B. T. Hallam and J. R. Sambles (unpublished).

[20] M. S. Bancroft, Ph.D. thesis, University of Manchester, 1991.

[21] T. J. Sluckin and A. Poniewierski, in Fluid Interfacial Phenomena, edited by C. A. Croxton (Wiley, London, 1985).

[22] S. Faetti, M. Gatti, V. Palleschi, and T. J. Sluckin, Phys. Rev. Lett. 55, 1681 (1985).

[23] A. A. Sonin, The Surface Physics of Liquid Crystals (Gordon and Breach, Amsterdam, 1995).

[24] I. Haller, H. A. Higgins, and T. R. McGuitre, J. Phys. Chem. 77, 950 (1973). 